\title{
Clinical importance of epicardial adipose tissue
}

\author{
Eszter Nagy, Adam L. Jermendy, Bela Merkely, Pal Maurovich-Horvat
}

MTA-SE Cardiovascular Imaging Research Group, Budapest, Hungary

Submitted: 4 June 2016

Accepted: 23 August 2016

Arch Med Sci 2017; 13, 4: 864-874

DOI: https://doi.org/10.5114/aoms.2016.63259

Copyright $\odot 2016$ Termedia \& Banach

\section{Abstract}

Different visceral fat compartments have several systemic effects and may play a role in the development of both insulin resistance and cardiovascular diseases. In the last couple of years special attention has been paid to the epicardial adipose tissue (EAT), which can be quantified by non-invasive cardiac imaging techniques. The epicardial fat is a unique fat compartment between the myocardium and the visceral pericardium sharing a common embryologic origin with the visceral fat depot. Epicardial adipose tissue has several specific roles, and its local effects on cardiac function are incorporated in the complex pathomechanism of coronary artery disease. Importantly, EAT may produce several adipocytokines and chemokines that may influence - through paracrine and vasocrine effects - the development and progression of coronary atherosclerosis. Epicardial adipose tissue volume has a relatively strong genetic dependence, similarly to other visceral fat depots. In this article, the anatomical and physiological as well as pathophysiological characteristics of the epicardial fat compartment are reviewed.

Key words: coronary artery disease, epicardial adipose tissue, insulin resistance syndrome, visceral fat compartments, epicardial fat.

\section{Introduction}

Type 2 diabetes mellitus due to its prevalence rate and cardiovascular complications carries a serious burden for health care systems worldwide [1]. Insulin resistance syndrome with the dysfunction of the abdominal fat compartment plays an important role in the disease development [2, 3]. In the last couple of years it was documented that other fat compartments may also be involved in the insulin resistance syndrome and may contribute to the pathogenesis of atherosclerosis [4]. Recently, special attention has been paid to the epicardial fat compartment [5].

In the $19^{\text {th }}$ century it was believed that fatty degeneration of the heart is the main cause of every heart disease [6]. Richard Quain was the most well-known proponent of this theory, recognizing the relationship of increased fat volume on the epicardial surface with coronary artery obstruction. The diagnosis of fatty heart was very popular in the Victorian era but was later changed to fibrosus heart disease and chronic myocarditis. All these diagnoses were replaced by the ischemia theory in the middle of the $20^{\text {th }}$ century. Interestingly, it was recognized at that time that $70 \%$ of the fatty heart diagnoses in Quain's pathological records corresponded with ischemic heart disease. Although the relationship between increased epicardial fat and cardiac diseases was described nearly 150 years ago, medicine did not dedicate too much attention to this

\author{
Corresponding author: \\ Pal Maurovich-Horvat PhD, \\ $\mathrm{MPH}$ \\ MTA-SE Cardiovascular \\ Imaging Research Group \\ Budapest, Hungary \\ 68 Varosmajor St \\ 1122 Budapest, Hungary \\ Phone: +36 203879193 \\ E-mail: maurovich.horvat@ \\ gmail.com
}


field. However, cardiovascular research has begun to explore the role of different fat compartments in line with the pandemic spread of obesity and the dynamic development of radiological imaging techniques [7]. In this regard, special attention was paid to the epicardial fat due to its anatomical proximity with the coronary arteries [8]. While anatomical and biochemical characteristics of the epicardial fat compartment were described in early studies, its potential role in the pathomechanism of coronary artery disease (CAD) and other cardiac dysfunctions has only been investigated recently.

In this article, the anatomical and physiological as well as pathophysiological characteristics of the epicardial fat compartment are reviewed.

\section{Terminology}

The terminology of fat compartments around the heart is not standardized; there are still many imprecise uses of these definitions in the literature. Nevertheless, the most widely used and accepted terms are summarized in Table I.

The epicardial fat as a part of the visceral fat is localized between the myocardial surface and the visceral layer of the pericardium. Pericardial fat involves adipose tissues between the two (visceral and parietal) pericardial layers and the fat depot on the external surface of the parietal pericardium. Paracardial fat contains fat deposits outside the parietal pericardium and therefore sometimes is called extra-pericardial intrathoracic fat. The coronary arteries are surrounded by the perivascular/pericoronary fat, irrespective of location. The term ectopic fat implies triglyceride deposits in non-adipose tissue of different organs such as myocardium, liver, pancreas, etc. [9].

The clear distinction of epicardial fat from pericardial fat is of great clinical importance [10]. In the embryological aspect they differ from each other. While the epicardial fat is similar to the visceral fat and originates from mesodermal cells, the pericardial fat has an ectodermal origin, similar to subcutaneous fat. Moreover, there is also a difference in the blood supply between these two fat compartments; the epicardial fat is supplied by the small myocardial coronary arteries, while the circulation of pericardial fat is provided from the thoracic vessels. The amount of epicardial and pericardial fat compartments as a percentage of total cardiac mass also differs (Table II).

\section{Cardiac imaging of epicardial adipose tissue}

Epicardial fat tissue can be visualized and quantified non-invasively by echocardiography, magnetic resonance imaging (MRI) and cardiac computed tomography (CT).

Echocardiography provides a simple, cheap and readily available assessment which directly pictures the epicardial adipose tissue (EAT) thickness on the free wall of the right ventricle. Imaging by echocardiography provides the parasternal shortand long-axis view in three consecutive end-systolic phases (Figure 1). Several studies have estab-

Table I. Terminology of fat compartments around the heart

\begin{tabular}{|c|c|}
\hline Visceral fat & Adipose tissue around the visceral organs \\
\hline Epicardial fat & $\begin{array}{c}\text { Visceral fat between the myocardial surface and the visceral layer } \\
\text { of the pericardium }\end{array}$ \\
\hline Pericardial fat & $\begin{array}{l}\text { Adipose tissue between the two pericardial layers (visceral and parietal } \\
\text { pericardium) and fat depot on the external surface of the parietal pericardium }\end{array}$ \\
\hline Paracardial fat & Fat deposits outside the parietal pericardium (extra-pericardial thoracic fat) \\
\hline Perivascular (pericoronary) fat & Adipose tissue around the vessels (coronary arteries) irrespective of location \\
\hline Ectopic fat & $\begin{array}{l}\text { Lipid (triglycerides) deposits in non-adipose tissue (i.e. myocardium, liver, } \\
\text { pancreas, etc.) }\end{array}$ \\
\hline
\end{tabular}

Table II. Differences between epicardial and pericardial fat compartments

\begin{tabular}{|lcc|}
\hline Variable & \multicolumn{1}{c|}{ Epicardial fat } & Pericardial fat \\
\hline Location & $\begin{array}{c}\text { Between the myocardial surface and } \\
\text { the visceral pericardium }\end{array}$ & $\begin{array}{c}\text { Outside the visceral pericardium, between } \\
\text { the visceral and parietal pericardium and } \\
\text { on the external surface of the parietal } \\
\text { pericardium }\end{array}$ \\
\hline Embryologic origin & Splanchnopleuric mesoderm & Primitive thoracic mesenchyme \\
\hline Blood supply & Branches from the coronary arteries & $\begin{array}{c}\text { Non-coronary sources (branches from the } \\
\text { internal mammary artery) }\end{array}$ \\
\hline Amount & $20 \%$ of total heart weight & $20-40 \%$ of cardiac mass \\
\hline
\end{tabular}




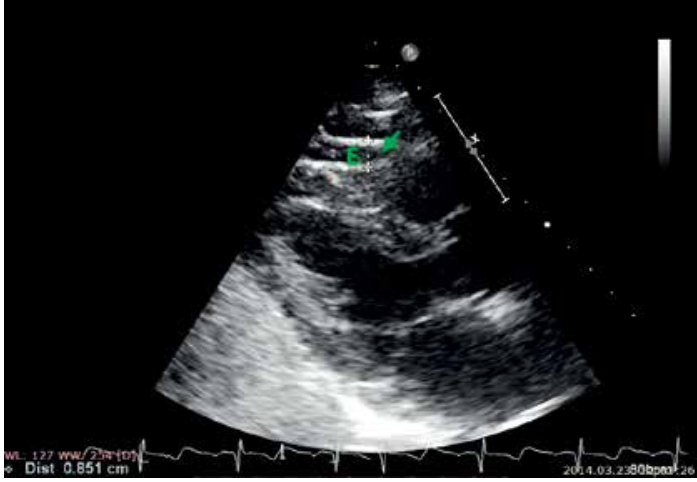

Figure 1. Quantification of epicardial adipose tissue by echocardiography (parasternal view). The thickness of the area between the myocardium and the visceral layer of the pericardium is $0.85 \mathrm{~cm}$, indicating epicardial adipose tissue

lished the general EAT thickness under $7 \mathrm{~mm}$ in the asymptomatic population [11]. Nevertheless, this method has several disadvantages including poor reproducibility and high dependence on the observer's experience. In addition, it may not reflect accurately the whole quantity of the epicardial fat due to the two-dimensional nature of the measurement. In other words, the thickness rather than the entire quantity of the pericardial fat compartment can be assessed by echocardiography. Moreover, the method has poor intra- and interobserver variability, and its results may differ significantly from the measurements with CT [12].

In contrast to echocardiography, MRI provides accurate area measurements with enhanced spinecho sequence and, in this way, EAT area mass and volume can be calculated (Figure 2). Area measurements with MRI correspond well with fat thickness determination with echocardiography, although the Bland-Altman analysis shows a systemic bias through overestimation of EAT with echocardiography [13]. Magnetic resonance imag-

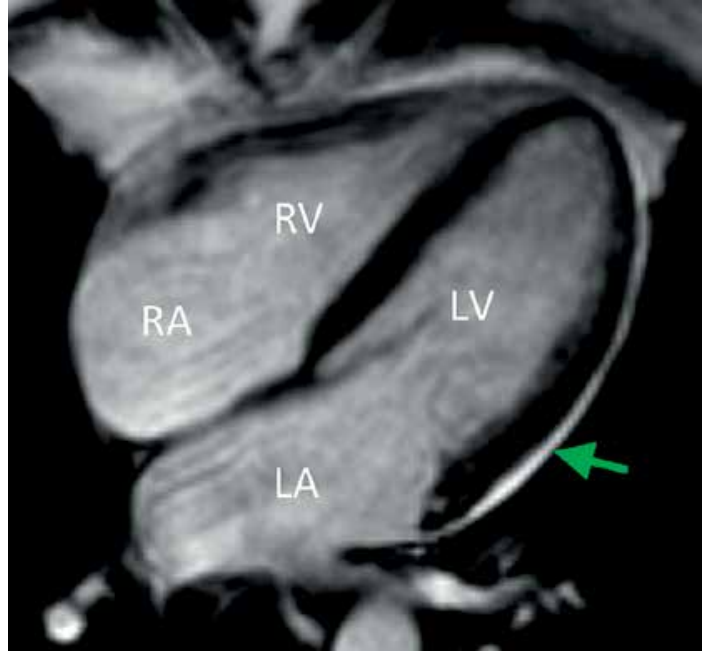

Figure 2. Epicardial adipose tissue (arrow) by using magnetic resonance imaging (MRI) technique

$R A$ - right atrium, $L A$ - left atrium, $R V$ - right ventricle, $L V$ - left ventricle.

ing is hardly available in routine clinical practice, is more expensive and has poorer spatial resolution compared to CT.

True volume assessment of EAT is feasible using cardiac CT. The three dimensional (3D) image reconstruction with multidetector-row $\mathrm{CT}$ (MDCT) has the best spatial resolution among the imaging modalities (Figure 3). It is of note that the specificity and sensitivity of measurements with MDCT are the best when compared to alternative imaging methods. The epicardial fat quantification is performed on prospectively ECG triggered non-contrast $\mathrm{CT}$ scans which extend from the pulmonary artery bifurcation to the diaphragm. The identification of the EAT is based on thresholds of CT attenuation. Typically, lower thresholds of CT attenuation range from -250 to -190 Hounsfield units (HU), and upper thresholds are
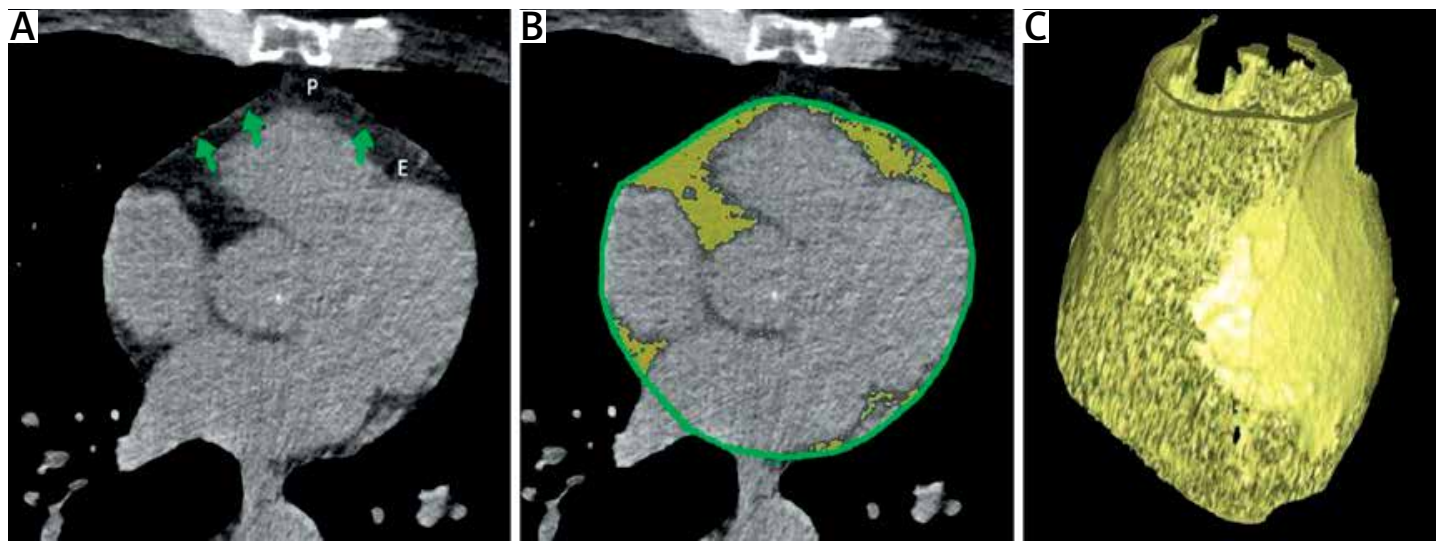

Figure 3. Measuring epicardial adipose tissue by cardiac computed tomography (CT). A - Axial section at the aortic root. Arrows indicate the visceral layer of the pericardium. Epicardial fat $(E)$ is located inside and pericardial fat $(P)$ outside the visceral layer. B - The visceral layer of the pericardium is traced manually (green). Epicardial adipose tissue (yellow) is marked automatically at the corresponding section. $\mathbf{C}$ - Three-dimensional reconstruction of the total epicardial fat compartment (yellow). The volume of epicardial adipose tissue was $112 \mathrm{~cm}^{3}$ 
set between -50 and $-30 \mathrm{HU}$. In contrast to area and thickness measurements, volume quantification provides the most accurate way for assessing the true epicardial fat quantity [14]. Using this method, coronary artery calcification may also be quantified, resulting in more reliable cardiovascular risk assessment [15]. Importantly, native CT results in a very small ( $1 \mathrm{mSv}$ ) radiation dose. Maurovich-Horvat et al. found in a collaborative study that the measurement of pericoronary adipose tissue was highly reproducible when using MDCT [16].

\section{Anatomical characteristics of epicardial adipose tissue}

In physiological circumstances the epicardial fat covers nearly $80 \%$ of the heart surface. According to previous observations this fat compartment contributes $20 \%$ to the whole heart quantity [17]. The EAT-covered heart region includes the heart base and the apex, the atrioventricular sulci, the entire surface of the right ventricle, and the great coronary vessels with their origins. The distribution of EAT is mostly inhomogeneous; the biggest mass is localized on the lateral and anterior walls of the right atrium, but in normal circumstances it also covers the atrioventricular and the interventricular sulci and the main coronary arteries as well. In the case of extremely enlarged EAT, it can also accumulate on the surface of the left atrium and along the vessel's adventitia with spreading into the myocardium. It is of note that there is no separating fascia layer between the epicardial fat and the myocardium, providing close proximity of these two different tissues [18]. In histological investigations it has been previously established that adipocytes in the EAT are smaller than those in the abdominal or the subcutaneous fat compartments [19]. Besides adipocytes, EAT includes nerves, ganglions, vessels, inflammatory cells and fibrocytes as well (Figure 4).

Age, gender, body weight and ethnicity should be taken into consideration among physiological determinants of EAT. Epicardial adipose tissue seems to increase with age [20]. The quantity of EAT depends on gender and body mass index (BMI). For example, pericardial fat was reported to be $137 \pm 54 \mathrm{~cm}^{3}$ among men and $108 \pm 41 \mathrm{~cm}^{3}$ among women of the Framingham offspring cohort [21]. In patients with a high BMI $\left(>27 \mathrm{~kg} / \mathrm{m}^{2}\right)$, EAT volume was more than two times higher compared to those with a BMI $<27 \mathrm{~kg} / \mathrm{m}^{2}\left(155 \pm 15 \mathrm{~cm}^{3} \mathrm{vs}\right.$. $67 \pm 12 \mathrm{~cm}^{3}$ ) [22]. Some ethnic differences in epicardial and pericardial fat thickness may also occur; non-Hispanic White men have more epicardial and pericardial fat than do African Americans [23].

The biochemical features of small adipocytes in EAT may also differ from those of other fat compartments. In experimental studies EAT had a higher rate of free fatty acid (FFA) release than adipose tissue elsewhere in the body, suggesting that EAT might play a role in local energy supply for the myocardium. In addition, a lower oxidative capacity and a lower rate of glucose utilization were also documented [24]. On the other hand, a 5-fold higher expression of uncoupled protein-1 (UCP-1) was found in EAT compared to other fat depots [25]. Uncoupled protein-1 is a specific protein in brown fat which is necessary for its energy production, and does not appear in other types of fat tissues. This latter feature is in line with the fact that epicardial fat evolves from brown adipose tissue during embryogenesis.

\section{Physiological function of epicardial adipose tissue}

Several physiological functions of EAT are already known from different studies or inferred from its biochemical or anatomical features. Unfortunately, experimental evidence supporting these observations are limited due to the very small amount of EAT in experimental animals (rodents).

It is suggested that functions of EAT may include protection of the myocardium against hypothermia [25]. In addition, EAT can provide a mechanical protective role for coronary circulation. It can attenuate the torsion developed by the myocardium contraction or the arterial pulse wave, but it has a permissive role as well in positive remodeling of coronary arteries [26].

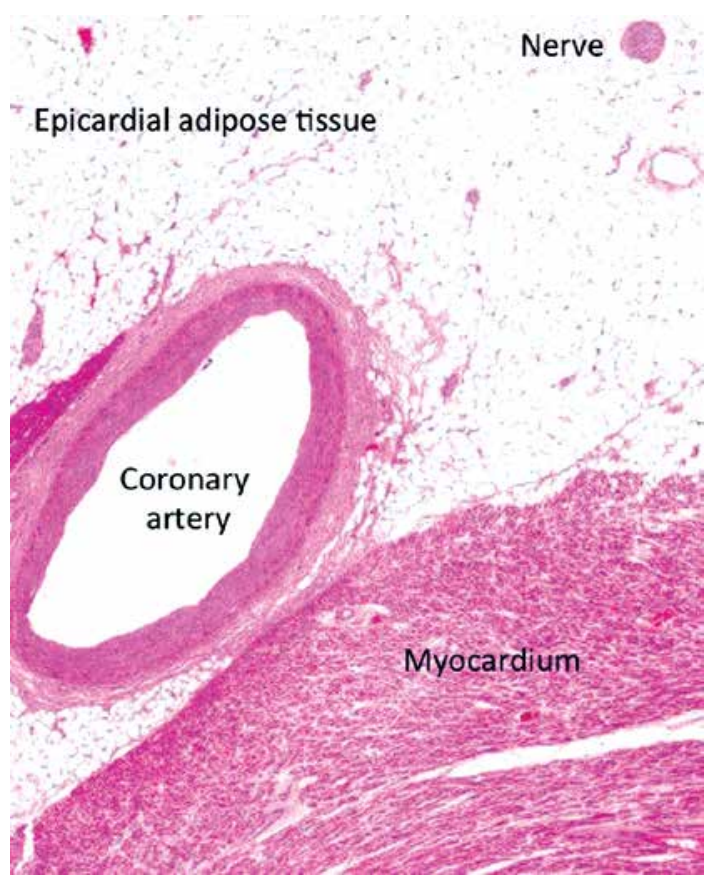

Figure 4. Microscopic view of the epicardial adipose tissue. It is of note that there is no separating fascia layer between the epicardial fat and the myocardium 
Also, EAT has a substantial role in energy supply to the myocardium and should be considered as a provider of energy during periods of high energy demand [27]. On the other hand, EAT may protect the myocardium from the cardiotoxic effect of a large amount of FFA due to its capacity for fast FFA utilization [28]. Taken together, EAT may serve as a unique energy buffering pool in the homeostasis of the myocardium.

In addition, adiponectin secretion from epicardial adipocytes may promote the coronary circulation. Adiponectin improves endothelial function through stimulation of nitrogen monoxide synthase, reduces oxidative stress, and indirectly decreases the level of interleukin-6 (IL-6) and C-reactive protein (CRP) by reducing tumor necrosis factor- $\alpha$ (TNF- $\alpha)$ production $[29,30]$. Adiponectin also has some extracardial effects such as increased glucose utilization in the hepatocytes and muscle cells which may result in improving insulin sensitivity [31].

\section{Epicardial adipose tissue in the pathomechanism of atherosclerosis}

Some years ago a hypothesis about the direct role of EAT in the development and progression of coronary atherosclerosis was raised, and paracrine and vasocrine effects of EAT due to close proximity of epicardial fat to coronary arteries were posited [32]. The hypothesis was indirectly supported by a pathological study in subjects with a myocardial bridge. Namely, no atherosclerosis was observed in coronary segments at the myocardial bridge where surrounding fat on the coronary arteries was lacking [33].

In a landmark study, Mazurek et al. analyzed epicardial and subcutaneous fat from the lower extremity in obese patients referred for coronary artery bypass grafting. They found increased lev- els of inflammatory mediators (IL-6, TNF- $\alpha$, inter-

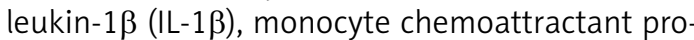
tein-1 (MCP-1)), macrophages, lymphocytes and basophils in epicardial fat as compared to subcutaneous fat compartments [34]. Others found that epicardial and omental fat exhibited a broadly comparable pathogenic messenger ribonucleotide acid (mRNA) profile indicating macrophage infiltration into the epicardial fat [35]. In another study, mediators of the nuclear factor- $\kappa \mathrm{B}(\mathrm{NF}-\kappa \mathrm{B})$ and c-Jun $\mathrm{N}$-terminal kinase (JNK) pathways were suggested to be involved in the inflammatory profile of EAT, highlighting the role of the macrophages in the inflammation within this tissue [36]. These studies indicate that chronic inflammation occurs locally as well as systemically, potentially contributing further to the pathogenesis of CAD.

It was documented that the epicardial adipocytes had impaired adiponectin secretion and in creased leptin production in obese patients with hypertension, metabolic syndrome and CAD [37, 38]. This shift in the adiponectin/leptin ratio enhances the development of atherosclerosis. Namely, the decreased adiponectin expression attenuates endothelial function and leads to increased TNF- $\alpha$ production, triggering systematic inflammation and oxidative stress. The altered leptin level promotes atherogenic changes in endothelial cells such as increased adhesion of monocytes, a higher level of macrophage-to-foam cell transformation, unfavorable changes in lipid levels, and elevation of CRP and inflammatory cytokine levels. All these alterations may lead to development and destabilization of atherosclerotic plaques in coronary arteries [5].

Based on several studies it became widely accepted that EAT should be considered as a source of inflammatory mediators that might directly influence the myocardium and coronary arteries (Figure 5). Two mechanisms of influence (paracrine

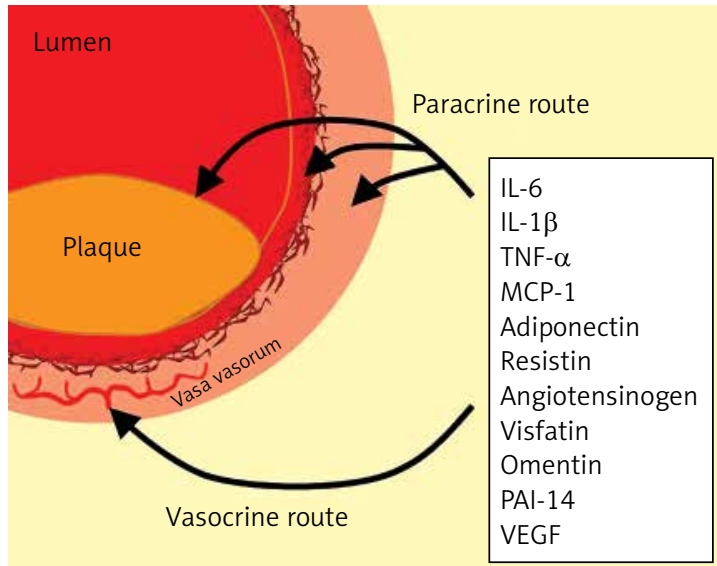

Figure 5. Routes for paracrine and vasocrine effects of epicardial adipose tissue on coronary arteries and plaque formation

IL - interleukin, TNF- $\alpha$ - tumor necrosis factor- $\alpha, M C P$ - 1 - monocyte chemoattractant protein-1, PAI-1 - plasminogen activator inhibitor-1,VEGF - vascular endothelial growth factor. 
and vasocrine) were suggested [39]. The paracrine way of influence means that adipokines released from pericoronary fat may diffuse across the arterial wall (adventitia, media, and intima) and finally can interact with endothelial cells in the intima and with vascular smooth cells in the media. The alternative vasocrine way of effect can be achieved by release of adipocytokines and FFAs from EAT directly into the vasa vasorum of the coronary arterial wall [40]. It was suggested that the vasocrine way of influence may be predominant over the paracrine effect in the case of more advanced atherosclerotic lesions where inflammatory mediators may diffuse only with difficulties [35].

The association between EAT thickness and the metabolic syndrome was documented in a recent meta-analysis [41]. The relationship of EAT with CAD has been analyzed by several clinical studies $[42,43]$. In the Framingham and the MESA (Multiethnic Study of Atherosclerosis) epidemiological studies a significant association of epicardial fat with coronary artery calcification was found, which remained significant after adjustment for traditional cardiovascular risk factors [44, 45]. The increased epicardial fat proved to be associated with more advanced atherosclerosis in another study [46]. Epicardial fat was associated with non-calcified coronary plaques as well [47, 48]. A significant relationship of increased epicardial fat volume (> $130.7 \mathrm{~cm}^{3}$ ) with vulnerable plaques was also documented [49]. The relationship of morphological features of vulnerable plaques (positive remodeling, spotty calcifications, and low CT attenuation in the necrotic core) to the pericardial fat was also studied, and the volume of pericardial fat proved to be nearly twice as high in patients with vulnerable plaques as compared to those without CAD [50]. Pericardial fat was associated with myocardial ischemia detected by single photon emission computed tomography (SPECT) in patients without known CAD [51]. Epicardial adipose tissue correlated with the degree of coronary atheromatosis, suggesting that its excessive accumulation might contribute to the development of acute coronary syndrome and coronary total occlusions [52]. In another study, EAT thickness was independently associated with the Thrombolysis in Myocardial Infarction (TIMI) risk score in patients with non-ST-elevation myocardial infarction (NSTEMI) and unstable angina pectoris [53]. In patients with the metabolic syndrome, increased EAT was associated with impaired coronary flow reserve [54]. In a different patient population (in women with chest pain and angiographically normal coronary arteries), EAT thickness was correlated with reduced coronary flow reserve [55]. Different surrogate parameters of atherosclerosis were also investigated by others, and an association between EAT thickness and carotid intima-media thickness in type 2 diabetic patients as well as in children and adolescents with obesity was found $[56,57]$. Moreover, EAT showed an independent association with arterial stiffness in an asymptomatic Korean cohort [58]. In a recent study, Maurovich-Horvat et al. investigated the relationship of different thoracic fat depots with coronary atherosclerosis and found an independent association between pericoronary fat and CAD. In addition, pericoronary fat correlated with inflammatory biomarkers as well, suggesting that while systemic inflammation plays a role in the pathogenesis of CAD, additional local effects may exist [59]. In a systematic review and meta-analysis, an association between the elevated location-specific thickness of EAT at the left atrioventricular groove and obstructive CAD was found [60].

\section{Epicardial adipose tissue and other cardiac abnormalities}

The relationship of EAT with atrial fibrillation was analyzed in several clinical studies. A strong association between EAT and atrial fibrillation (both paroxysmal and persistent) was documented by Al Chekakie et al.; the relationship proved to be independent of traditional risk factors and atrial enlargement [61]. In another study, EAT thickness was verified as an independent predictor for post-ablative recurrence of atrial fibrillation [62]. In patients with peritoneal dialysis, increased EAT was associated with impaired left ventricle diastolic capacity independently of CRP level, a marker of systemic inflammation [63].

\section{Epicardial adipose tissue necrosis: a benign cause of chest pain}

Epicardial fat necrosis is a rare clinical condition; 26 cases were reported up to 2011 [64]. It should be considered in the differential diagnosis of chest pain. The etiology is obscure, but the prognosis is good. In general, the presenting symptom is left-sided chest pain in a previously healthy individual with an associated juxtacardiac mass seen in chest radiography. The CT or MRI may confirm the correct diagnosis, resulting in the avoidance of surgical intervention.

\section{Epicardial adipose tissue in type 2 diabetes, obesity and the insulin resistance syndrome (metabolic syndrome)}

Typically, type 2 diabetes is preceded by prediabetes, but insulin resistance syndrome due to obesity may be the first pathological stage in the long-lasting asymptomatic period of diabetes. The insulin resistance syndrome (also called the metabolic syndrome) includes insulin resistance and 
different metabolic abnormalities (elevated serum triglycerides, lower HDL cholesterol, hyperglycemia) as well as elevated blood pressure. Obesity, especially abdominal visceral fat accumulation, plays a central role in this syndrome. Although the use of the term and the suggested pathomechanism of the metabolic syndrome became debatable some years ago, the association between an enlarged abdominal visceral fat compartment and increased cardiovascular risk remained unquestionable [65]. The enlarged visceral fat depot is characterized primarily by increased lipolysis leading to hepatic steatosis. Non-alcoholic fatty liver disease (NAFLD) is often regarded as the hepatic manifestation of insulin resistance [66] and is considered as a novel predictor of cardiovascular disease $[67,68]$.

Several clinical investigations have aimed to assess the characteristics of EAT in the metabolic syndrome, prediabetes and type 2 diabetes. In a meta-analysis, EAT was $7.5 \pm 0.1 \mathrm{~mm}$ in thickness in the metabolic syndrome $(n=427)$ compared to $4.0 \pm 0.1 \mathrm{~mm}$ in controls $(n=301)$, and EAT correlated significantly with the components of the metabolic syndrome [69]. Epicardial adipose tissue volume was significantly higher in patients with type 2 diabetes than in nondiabetic subjects, and EAT volume was significantly associated with components of the metabolic syndrome [46]. In asymptomatic type 2 diabetic patients the thickness of EAT proved to be an independent risk factor for significant coronary artery stenosis but not for silent myocardial ischemia [70]. A strong correlation was found between fasting plasma glucose and EAT measured with CT or echocardiography [71, 72]. Epicardial adipose tissue quantity was higher in patients with type 2 diabetes mellitus compared to lean subjects or obese patients without diabetes. In addition, the difference in EAT volume between men and women was more pronounced in subjects with impaired fasting glucose or diabetes mellitus [73]. A clear relationship of epicardial fat and serum alanine aminotransferase (ALT) and aspartate aminotransferase (AST) activity, surrogate markers of fatty liver, was documented in a cross-sectional, observational study [74]. Taken together, the insulin resistance syndrome (the metabolic syndrome), type 2 diabetes, NAFLD and CAD are associated with an increased amount of epicardial fat [75].

In the majority of studies an increase of EAT volume was associated with stenosis of the coronary arteries [76, 77]. Since these studies are cross-sectional, it is uncertain whether adipose tissue plays a causal role in the development of atherosclerosis. Importantly, two longitudinal studies have reported results that support the hypothesis of 'outside to inside signaling' as a cause of atherosclerosis [45, 78]. In these studies, intrathoracic and EAT volume were measured and an increase of the quantity of intrathoracic and EAT was associated with incident coronary heart disease and with major adverse cardiac events. Associations were independent from BMI and other risk factors, suggesting that EAT is one of the factors contributing to CAD.

\section{Epicardial adipose tissue in type 1 diabetes}

Interestingly, higher epicardial fat and serum leptin levels were found in subjects with type 1 diabetes than in non-diabetic controls. The epicardial fat thickness and serum leptin levels proved to be the best independent correlates of each other in patients with type 1 diabetes independently of BMI, glycemic control and daily insulin requirement [79]. Recently, patients with type 1 diabetes $(n=100)$ from the Diabetes Control and Complications Trial/Epidemiology of Diabetes Interventions and Complications (DCCT/EDIC) study were investigated. In this pilot study, the accumulation of adipose tissue in epicardial and intra-thoracic spaces was strongly associated with higher BMI, greater waist-to-hip ratio, higher weighted glycated hemoglobin values, elevated triglycerides and a history of elevated albumin excretion rate or end-stage renal disease [80].

\section{Role of genetic effects on epicardial adipose tissue}

As EAT and other abdominal fat compartments (subcutaneous adipose tissue (SAT), visceral adipose tissue (VAT), hepatic lipid accumulation) carry different clinical significance [81] and epicardial fat differs from pericardial fat from an embryological point of view, the role of genetic effects on EAT and other fat compartments may differ. In a classical twin study with CT investigation our preliminary results indicated that EAT had a relatively strong genetic dependence, similarly to BMI and waist circumference $[82,83]$. In contrast to abdominal fat compartment areas, a weak genetic and a stronger environmental dependence of hepatic lipid accumulation was found in our twin cohort [84].

\section{Treatment options for modifying epicardial adipose tissue volume}

Lifestyle changes, bariatric surgery and various drugs may be applied. Reduction in weight (BMI) by using a very-low calorie diet or exercise training program in obese patients is associated with a decrease in EAT volume $[85,86]$. Nevertheless, this was observed after bariatric surgery as well, although myocardial triglyceride content did not change significantly [87]. In a meta-analysis, diet 
or bariatric surgery proved to be more beneficial than exercise training in reducing EAT volume [88]. The effect of drugs on EAT is controversial. Atorvastatin resulted in a more pronounced decrease of EAT than simvastatin/ezetimibe [89]. Pioglitazone compared with metformin increased pericardial fat volume in patients with type 2 diabetes [90]. Short-term (3 months) use of glucagon-like-receptor agonists (exenatide, liraglutide) decreased the volume of EAT in type 2 diabetic patients [91]. In a longer (26 weeks) randomized controlled trial, exenatide twice daily (versus standard antidiabetic treatment) proved to be effective in reducing both epicardial and liver fat content in obese patients with type 2 diabetes; the effects were mainly weight loss dependent [92]. In another study, sitagliptin, a dipeptidyl-peptidase-4 inhibitor, also decreased the volume of EAT in a 24-week long study with obese type 2 diabetic patients [93]. Clearly, EAT should be considered as a novel therapeutic target, and statins, pioglitazone as well as incretin-based drugs are the best candidates so far $[94,95]$.

\section{Conclusions}

The epicardial fat is a unique fat compartment located between the myocardial surface and the visceral layer of the pericardium. The EAT can be quantified by non-invasive cardiac imaging techniques such as echocardiography, MRI or cardiac CT.

Among physiological determinants of EAT, age, gender, body weight and ethnicity should be considered. The EAT volume has a relatively strong genetic dependence, similarly to other visceral fat depots. Physiological functions of EAT may include protection of the myocardium against hypothermia and a mechanical protective role for coronary circulation. In addition, EAT may serve as a unique energy buffering pool in the homeostasis of the myocardium.

As for pathophysiological functions, it is widely accepted that EAT should be considered as a source of inflammatory mediators that might directly influence the myocardium and coronary arteries. In line with these observations, clinical studies suggested that EAT - through paracrine and vasocrine effects - may have an impact on the development and progression of coronary atherosclerosis. In addition, an association between increased EAT and atrial fibrillation was also documented. The insulin resistance syndrome (the metabolic syndrome), type 2 diabetes, NAFLD and CAD proved to be associated with an increased amount of epicardial fat. Interestingly, accumulation of EAT was also observed in patients with type 1 diabetes.

Treatment options for modifying EAT volume include lifestyle changes, bariatric surgery and using different drugs. Weight reduction in obese subjects may lead to a decrease in EAT volume, while effects of different drugs on EAT are controversial. Nevertheless, EAT should be considered as a new cardiovascular therapeutic target.

\section{Acknowledgments}

A grant from the New Horizons Programme (European Foundation for the Study of Diabetes) is acknowledged. The microscopic imaging of human myocardium and epicardial fat was provided by Zoltán Sápi MD, DSc, Semmelweis University, Institute of Pathology, Budapest.

Eszter Nagy and Adam L. Jermendy equally contributed to this manuscript.

\section{Conflict of interest}

The authors declare no conflict of interest.

\section{References}

1. Zimmet P, Alberti KGMM, Shaw J. Global and societal implications of diabetes epidemic. Nature 2001; 414: 782-7.

2. Despres JP, Lemieux I. Abdominal obesity and metabolic syndrome. Nature 2006; 444: 881-7.

3. Poirier P, Despres JP. Waist circumference, visceral obesity, and cardiovascular risk. J Cardiopulmonary Rehabil 2003; 23: 161-9.

4. Lim S, Meigs JB. Links between ectopic fat and vascular disease in humans. Arterioscler Thromb Vasc Biol 2014; 34: 1820-6.

5. lacobellis G, Malavazos AE, Corsi MM. Epicardial fat: from biomolecular aspects to the clinical practice. Int J Biochem Cell Biol 2011; 43: 1651-4.

6. Bedford E. The story of fatty heart. A disease of Victorian times. Br Heart J 1972; 34: 23-8.

7. Després JP, Cartier A, Côté M, Arsenault BJ. The concept of cardiometabolic risk: bridging the fields of diabetology and cardiology. Ann Med 2008; 40: 514-23.

8. Iacobellis G, Ribaudo MC, Assael F, et al. Echocardiographic epicardial adipose tissue is related to anthropometric and clinical parameters of metabolic syndrome: a new indicator of cardiovascular risk. J Clin Endocrinol Metab 2003; 88: 5163-8.

9. lacobellis G, Bianco AC. Epicardial adipose tissue: emerging physiological, pathophysiological and clinical features. Trends Endocrinol Metab 2011; 22: 450-7.

10. lacobellis G. Epicardial and pericardial fat: close, but very different. Obesity 2009; 17: 625.

11. lacobellis G, Willens HJ. Echocardiographic epicardial fat: a review of research and clinical applications. J Am Soc Echocardiogr 2009; 22: 1311-9.

12. Saura D, Oliva MJ, Rodríguez D, et al. Reproducibility of echocardiographic measurements of epicardial fat thickness. Int J Cardiol 2010; 141: 311-3.

13. Sicari $R$, Sironi $A M$, Petz $R$, et al. Pericardial rather than epicardial fat is a cardiometabolic risk marker: an MRI vs echo study. J Am Soc Echocardiogr 2011; 24: 1156-62.

14. Gorter PM, van Lindert AS, de Vos AM, et al. Quantification of epicardial and peri-coronary fat using cardiac computed tomography; reproducibility and relation with obesity and metabolic syndrome in patients sus- 
pected of coronary artery disease. Atherosclerosis 2008; 197: 896-903.

15. Madaj P, Budoff MJ. Risk stratification of non-contrast CT beyond the coronary calcium scan. J Cardiovasc Comput Tomogr 2012; 6: 301-7.

16. Maurovich-Horvat P, Kallianos K, Engel LC, et al. Influence of pericoronary adipose tissue on local coronary atherosclerosis as assessed by a novel MDCT volumetric method. Atherosclerosis 2011; 219: 151-7.

17. Rabkin SW. Epicardial fat: properties, function and relationship to obesity. Obes Rev 2007; 8: 253-61.

18. lacobellis G, Corradi D, Sharma AM. Epicardial adipose tissue: anatomic, biomolecular and clinical relationships with the heart. Nat Clin Pract Cardiovasc Med 2005; 2: 536-43.

19. Bambace C, Telesca M, Zoico E, et al. Adiponectin gene expression and adipocyte diameter: a comparison between epicardial and subcutaneous adipose tissue in men. Cardiovasc Pathol 2011; 20: e153-6.

20. Bertaso AG, Bertol D, Duncan BB, Foppa M. Epicardial fat: definition, measurements and systematic review of main outcomes. Arq Bras Cardiol 2013; 101: e18-28.

21. Fox CS, Gona P, Hoffmann U, et al. Pericardial fat, intrathoracic fat, and measures of left ventricular structure and function: the Framingham Heart Study. Circulation 2009; 119: 1586-91.

22. Gorter PM, van Lindert AS, de Vos AM, et al. Quantification of epicardial and peri-coronary fat using cardiac computed tomography; reproducibility and relation with obesity and metabolic syndrome in patients sus pected of coronary artery disease. Atherosclerosis 2008; 197: 896-903.

23. Willens HJ, Gómez-Marín O, Chirinos JA, Goldberg R Lowery $\mathrm{MH}$, lacobellis $\mathrm{G}$. Comparison of epicardial and pericardial fat thickness assessed by echocardiography in African American and non-Hispanic White men: a pilot study. Ethn Dis 2008; 18: 311-6.

24. Marchington JM, Mattacks CA, Pond CM. Adipose tissue in the mammalian heart and pericardium: structure, foetal development and biochemical properties. Comp Biochem Physiol B 1989; 94: 225-32.

25. Sacks HS, Fain JN, Holman B, et al. Uncoupling protein-1 and related messenger ribonucleic acids in human epicardial and other adipose tissues: epicardial fat functioning as brown fat. J Clin Endocrinol Metab 2009; 94 3611-5.

26. Prati F, Arbustini E, Labellarte A, et al. Eccentric atherosclerotic plaques with positive remodelling have a pericardial distribution: a permissive role of epicardial fat? A three-dimensional intravascular ultrasound study of left anterior descending artery lesions. Eur Heart J 2003; 24: 329-36.

27. lacobellis G, Bianco AC. Epicardial adipose tissue: emerging physiological, pathophysiological and clinical features. Trends Endocrinol Metab 2011; 22: 450-7.

28. lozzo P. Metabolic toxicity of the heart: insights from molecular imaging. Nutr Metab Cardiovasc Dis 2010; 20: 147-56.

29. Li R, Wang WO, Zhang $\mathrm{H}$, et al. Adiponectin improves endothelial function in hyperlipidemic rats by reducing oxidative/nitrative stress and differential regulation of eNOS/iNOS activity. Am J Physiol Endocrinol Metab 2007; 293: E1703-8.

30. Deng G, Long Y, Yu YR, Li MR. Adiponectin directly improves endothelial dysfunction in obese rats through the AMPK-eNOS pathway. Int J Obes (Lond) 2010; 34: 165-71.
31. Payne GA, Kohr MC, Tune JD. Epicardial perivascular adipose tissue as a therapeutic target in obesity-related coronary artery disease. Br J Pharmacol 2012; 165: 659-69.

32. Sacks HS, Fain JN. Human epicardial adipose tissue: a review. Am Heart J 2007; 153: 907-17.

33. Ishii T, Asuwa N, Masuda S, Ishikawa Y. The effects of a myocardial bridge on coronary atherosclerosis and ischaemia. J Pathol 1998; 185: 4-9.

34. Mazurek T, Zhang L, Zalewski A, et al. Human epicardial adipose tissue is a source of inflammatory mediators. Circulation 2003; 108: 2460-6.

35. Baker AR, Silva NF, Quinn DW, et al. Human epicardial adipose tissue expresses a pathogenic profile of adipocytokines in patients with cardiovascular disease. Cardiovasc Diabetol 2006; 5: 1.

36. Baker AR, Harte AL, Howell N, et al. Epicardial adipose tissue as a source of nuclear factor-kappaB and c-Jun $\mathrm{N}$-terminal kinase mediated inflammation in patients with coronary artery disease. J Clin Endocrinol Metab 2009; 94: 261-7.

37. Eiras S, Teijeira-Fernández E, Shamagian LG, Fernan$\operatorname{dez}$ AL, Vazquez-Boquete A, Gonzalez-Juanatey JR. Extension of coronary artery disease is associated with increased IL-6 and decreased adiponectin gene expression in epicardial adipose tissue. Cytokine 2008; 43: 174-80.

38. lacobellis G, Pistilli D, Gucciardo M, et al. Adiponectin expression in human epicardial adipose tissue in vivo is lower in patients with coronary artery disease. Cytokine 2005; 29: 251-5.

39. Sacks HS, Fain JN. Human epicardial adipose tissue: a review. Am Heart J 2007; 153: 907-17.

40. Yudkin JS, Eringa E, Stehouwer CD. "Vasocrine" signalling from perivascular fat: a mechanism linking insulin resistance to vascular disease. Lancet 2005; 365: 1817-20.

41. Pierdomenico SD, Pierdomenico AM, Cuccurullo F, lacobellis G. Meta-analysis of the relation of echocardiographic epicardial adipose tissue thickness and the metabolic syndrome. Am J Cardiol 2013; 111: 73-8.

42. Yerramasu A, Dey D, Venuraju S, et al. Increased volume of epicardial fat is an independent risk factor for accelerated progression of sub-clinical coronary atherosclerosis. Atherosclerosis 2012; 220: 223-30.

43. Wang TD, Lee WJ, Shih FY, et al. Association of epicardial adipose tissue with coronary atherosclerosis is region-specific and independent of conventional risk factors and intra-abdominal adiposity. Atherosclerosis 2010; 213: 279-87.

44. Rosito GA, Massaro JM, Hoffmann U, et al. Pericardial fat, visceral abdominal fat, cardiovascular disease risk factors, and vascular calcification in a community-based sample: the Framingham Heart Study. Circulation 2008; 117: 605-13.

45. Ding J, Hsu FC, Harris TB, et al. The association of pericardial fat with incident coronary heart disease: the Multi-Ethnic Study of Atherosclerosis (MESA). Am J Clin Nutr 2009; 90: 499-504.

46. Wang CP, Hsu HL, Hung WC, et al. Increased epicardial adipose tissue (EAT) volume in type 2 diabetes mellitus and association with metabolic syndrome and severity of coronary atherosclerosis. Clin Endocrinol (Oxf) 2009; 70: 876-82

47. Konishi M, Sugiyama S, Sugamura K, et al. Association of pericardial fat accumulation rather than abdomina obesity with coronary atherosclerotic plaque formation in patients with suspected coronary artery disease. Atherosclerosis 2010; 209: 573-8. 
48. Alexopoulos N, McLean DS, Janik M, Arepalli CD, Stillman AE, Raggi P. Epicardial adipose tissue and coronary artery plaque characteristics. Atherosclerosis 2010; 210: 150-4.

49. Ito T, Nasu K, Terashima M, et al. The impact of epicardial fat volume on coronary plaque vulnerability: insight from optical coherence tomography analysis. Eur Heart J Cardiovasc Imaging 2012; 13: 408-15.

50. Schlett CL, Ferencik M, Kriegel MF, et al. Association of pericardial fat and coronary high-risk lesions as determined by cardiac CT. Atherosclerosis 2012; 222: 129-34

51. Tamarappoo B, Dey D, Shmilovich H, et al. Increased pericardial fat volume measured from noncontrast $C T$ predicts myocardial ischemia by SPECT. JACC CardiovasC Imaging 2010; 3: 1104-12.

52. Ueno K, Anzai T, Jinzaki M, et al. Increased epicardial fat volume quantified by 64-multidetector computed tomography is associated with coronary atherosclerosis and totally occlusive lesions. Circ J 2009; 73: 1927-33.

53. Ozcan F, Turak O, Canpolat U, et al. Association of epicardial fat thickness with TIMI risk score in NSTEMI/USAP patients. Herz 2014; 39: 755-60.

54. Tok D, Çağli K, Kadife I, et al. Impaired coronary flow reserve is associated with increased echocardiographic epicardial fat thickness in metabolic syndrome patients. Coron Artery Dis 2013; 24: 191-5.

55. Sade LE, Eroglu S, Bozbaş $\mathrm{H}$, et al. Relation between epicardial fat thickness and coronary flow reserve in women with chest pain and angiographically normal coronary arteries. Atherosclerosis 2009; 204: 580-5.

56. Cetin M, Cakici M, Polat M, Suner A, Zencir C, Ardic I. Relation of epicardial fat thickness with carotid intimamedia thickness in patients with type 2 diabetes mellitus. Int J Endocrinol 2013; 2013: 769175.

57. Cabrera-Rego JO, lacobellis G, Castillo-Herrera JA, et al. Epicardial fat thickness correlates with carotid intima-media thickness, arterial stiffness, and cardiac geometry in children and adolescents. Pediatr Cardiol 2014; 35: 450-6.

58. Park HE, Choi SY, Kim HS, Kim MK, Cho SH, Oh BH. Epicardial fat reflects arterial stiffness: assessment using 256-slice multidetector coronary computed tomography and cardio-ankle vascular index. J Atheroscler Thromb 2012; 19: 570-6.

59. Maurovich-Horvat P, Kallianos K, Engel LC, et al. Relationship of thoracic fat depots with coronary atherosclerosis and circulating inflammatory biomarkers. Obesity (Silver Spring) 2015; 23: 1178-84.

60. Wu FZ, Chou KJ, Huang YL, Wu MT. The relation of location-specific epicardial adipose tissue thickness and obstructive coronary artery disease: systemic review and meta-analysis of observational studies. BMC Cardiovasc Disord 2014; 14: 62

61. Al Chekakie MO, Welles CC, Metoyer R, et al. Pericardial fat is independently associated with human atrial fibrillation. J Am Coll Cardiol 2010; 56: 784-8.

62. Chao TF, Hung CL, Tsao HM, et al. Epicardial adipose tissue thickness and ablation outcome of atrial fibrillation. PLoS One 2013; 8: e74926.

63. Lin HH, Lee JK, Yang CY, Lien YC, Huang JW, Wu CK. ACcumulation of epicardial fat rather than visceral fat is an independent risk factor for left ventricular diastolic dysfunction in patients undergoing peritoneal dialysis. Cardiovasc Diabetol 2013; 12: 127.

64. Baig A, Campbell B, Russell M, Singh J, Borra S. Epicardial fat necrosis: an uncommon etiology of chest pain. Cardiol J 2012; 19: 424-8.
65. Borch-Johnsen K, Wareham N. The rise and fall of the metabolic syndrome. Diabetologia 2010; 53: 597-9.

66. Abdelmalek MF, Diehl AM. Nonalcoholic fatty liver disease as a complication of insulin resistance. Med Clin North Am 2007; 91: 1125-49.

67. Hamaguchi M, Kojima T, Takeda N, et al. Nonalcoholic fatty liver disease is a novel predictor of cardiovascular disease. World J Gastroenterol 2007; 13: 1579-84.

68. Schindhelm RK, Diamant M, Heine RJ. Nonalcoholic fatty liver disease and cardiovascular disease risk. Curr Diab Rep 2007; 7: 181-7.

69. Rabkin SW. The relationship between epicardial fat and indices of obesity and the metabolic syndrome: a systematic review and meta-analysis. Metab Syndr Relat Disord 2014; 12: 31-42.

70. Kim HM, Kim KJ, Lee HJ, et al. Epicardial adipose tissue thickness is an indicator for coronary artery stenosis in asymptomatic type 2 diabetic patients: its assessment by cardiac magnetic resonance. Cardiovasc Diabetol 2012; 11: 83.

71. Wang TD, Lee WJ, Shih FY, et al. Relations of epicardial adipose tissue measured by multidetector computed tomography to components of the metabolic syndrome are region-specific and independent of anthropometric indexes and intraabdominal visceral fat. J Clin Endocrinol Metab 2009; 94: 662-9.

72. lacobellis G, Barbaro G, Gerstein HC. Relationship of epicardial fat thickness and fasting glucose. Int J Cardiol 2008; 128: 424-6.

73. lozzo P, Lautamaki R, Borra R, et al. Contribution of glucose tolerance and gender to cardiac adiposity. J Clin Endocrinol Metab 2009; 94: 4472-82.

74. Iacobellis G, Pellicelli AM, Grisorio B, et al. Relation of epicardial fat and alanine aminotransferase in subjects with increased visceral fat. Obesity (Silver Spring) 2008; 16: $179-83$.

75. lacobellis G. Local and systemic effects of the multifaceted epicardial adipose tissue depot. Nat Rev Endocrinol 2015; 11: 363-71.

76. Picard FA, Gueret P, Laissy JP, et al. Epicardial adipose tissue thickness correlates with the presence and severity of angiographic coronary artery disease in stable patients with chest pain. PLoS One 2014; 9: e110005.

77. Sinha SK, Thakur R, Jha MJ, et al. Epicardial adipose tissue thickness and its association with the presence and severity of coronary artery disease in clinical setting: a cross-sectional observational study. J Clin Med Res 2016; 8: 410-9.

78. Cheng VY, Dey D, Tamarappoo B, et al. Pericardial fat burden on ECG-gated noncontrast CT in asymptomatic patients who subsequently experience adverse cardiovascular events. JACC Cardiovasc Imaging 2010; 3: 352-60.

79. lacobellis G, Diaz S, Mendez A, Goldberg R. Increased epicardial fat and plasma leptin in type 1 diabetes independently of obesity. Nutr Metab Cardiovasc Dis 2014; 24: 725-9.

80. Darabian S, Backlund JY, Cleary PA, et al. Significance of epicardial and intrathoracic adipose tissue volume among type 1 diabetes patients in the DCCT/EDIC: a pilot study. PLoS One 2016; 11: e0159958.

81. Lim S, Meigs JB. Links between ectopic fat and vascular disease in humans. Arterioscler Thromb Vasc Biol 2014; 34: 1820-6.

82. Maurovich-Horvat P, Tárnoki DL, Tárnoki ÁD, et al. Rationale, design and methodological aspects of the BUDAPEST-GLOBAL study (Burden of Atherosclerotic Plaques 
Study in Twins - Genetic Loci and the Burden of Atherosclerotic Lesions). Clin Cardiol 2015; 38: 699-707.

83. Maurovich-Horvat P, Jermendy AL, Horcsik DV, et al. Epicardial adipose tissue quantity shows a relatively strong genetic dependence: a classic twin study (meeting abstract). Diabetes 2015; 64 Suppl 1: A111.

84. Jermendy AL, Drobni Z, Horvath T, et al. Hepatic lipid accumulation is mainly determined by environmental factors: a classic twin study (meeting abstract). Diabetes 2015; 64 Suppl 1: A554.

85. lacobellis G, Singh N, Wharton S, Sharma AM. Substantial changes in epicardial fat thickness after weight loss in severely obese subjects. Obesity (Silver Spring) 2008; 16: 1693-7.

86. Kim MK, Tomita T, Kim MJ, Sasai H, Maeda S, Tanaka K. Aerobic exercise training reduces epicardial fat in obese men. J Appl Physiol 2009; 106: 5-11.

87. Gaborit B, Jacquier A, Kober F, et al. Effects of bariatric surgery on cardiac ectopic fat: lesser decrease in epicardial fat compared to visceral fat loss and no change in myocardial triglyceride content. J Am Coll Cardiol 2012; 60: 1381-9.

88. Rabkin SW, Campbell H. Comparison of reducing epicardial fat by exercise, diet or bariatric surgery weight loss strategies: a systematic review and meta-analysis. Obes Rev 2015; 16: 406-15.

89. Park JH, Park YS, Kim YJ, et al. Effects of statins on the epicardial fat thickness in patients with coronary artery stenosis underwent percutaneous coronary intervention: comparison of atorvastatin with simvastatin/ezetimibe. J Cardiovasc Ultrasound 2010; 18: 121-6.

90. Jonker JT, Lamb HJ, van der Meer RW, et al. Pioglitazone compared with metformin increases pericardial fat volume in patients with type 2 diabetes mellitus. J Clin Endocrinol Metab 2010; 95: 456-60.

91. Morano S, Romagnoli E, Filardi T, et al. Short-term effects of glucagon-like peptide 1 (GLP-1) receptor agonists on fat distribution in patients with type 2 diabetes mellitus: an ultrasonography study. Acta Diabetol 2015; 52: 727-32.

92. Dutour A, Abdesselam I, Ancel P, et al. Exenatide decreases liver fat content and epicardial adipose tissue in patients with obesity and type 2 diabetes: a prospective randomised clinical trial using magnetic resonance imaging and spectroscopy. Diabetes Obes Metab 2016; 18: 882-91.

93. Lima-Martínez MM, Paoli M, Rodney M, et al. Effect of sitagliptin on epicardial fat thickness in subjects with type 2 diabetes and obesity: a pilot study. Endocrine 2016; 51: 448-55.

94. Mazurek T, Opolski G. Pericoronary adipose tissue: a novel therapeutic target in obesity-related coronary atherosclerosis. J Am Coll Nutr 2015; 34: 244-54.

95. lacobellis G. Epicardial fat: a new cardiovascular therapeutic target. Curr Opin Pharmacol 2016; 27: 13-8. 